\title{
La prescripción del ejercicio físico en el niño y el adolescente
}

\author{
Jorge Correa*
}

\section{Resumen}

El ejercicio físico tiene beneficios en cualquier etapa de la vida; sin embargo, la prescripción de éste varía, más si se tienen en cuenta las diferencias morfofisiológicas de los sujetos sobre todo en las primeras etapas del ciclo vital. El presente artículo tiene como objetivo presentar los resultados de la revisión documental, alrededor de los lineamientos generales propuestos en la literatura científica, en cuanto a los principios de la prescripción aplicados al niño y al adolescente, describiendo aspectos distintivos de su condición en cada etapa, de los beneficios del ejercicio físico regular, de la evaluación del fitness físico y de las recomendaciones para la prescripción.

Palabras clave: niños, adolescentes, prescripción del ejercicio, ejercicio físico.

\section{Abstract}

The physical exercise has benefits in any stage of the life, nevertheless the prescription of this varies, more if it mainly considers the morpho-physiology differences of the subjects in the first stages of the vital cycle. The present I articulate must like objective present/display the results of the documentary revision, around the proposed general lineaments in scientific Literature, as far as the principles of the prescription applied to the boy and the adolescent, describing distinguishing aspects of its condition in each stage, of the benefits of the regular physical exercise, of the evaluation of fitness physical and the recommendations for the prescription.

Key word: children, adolescents, prescription of the exercise, exercise.

\footnotetext{
* Universidad Colegio Mayor de Nuestra Señora del Rosario. Facultad de Rehabilitación y Desarrollo Humano. Bogotá. Colombia.
}

E, F.T, SP, Candidato a Magíster en Fisiología. Doctorando en Educación. Profesor de carrera, miembro del grupo de investigación en Ejercicio Físico y Desarrollo Humano. jecorrea@urosario.edu.co 


\section{Introducción}

Hoy por hoy, los niños y adolescentes son expuestos a patrones de vida sedentarios, asociados a la hipocinética dentro de las actividades del tiempo libre permitiendo la instauración de enfermedades crónicas no transmisibles en edades cada vez más tempranas (Rowland, 1990). En este sentido, varios estudios han demostrado que los niños activos, presentan niveles de aptitud física mejores lo que impacta de manera directa sobre su condición de salud (Sallis, 2000).

El ejercicio físico realizado de manera regular a edades tempranas cada vez más tempranas ha demostrado beneficios positivos para los procesos de crecimiento y desarrollo, pero no se ha evidenciado claramente el efecto protectivo que éste pueda tener, sobre la instauración de enfermedades crónicas no transmisibles, debido a que es difícil hacer un seguimiento a los estilos de vida inadecuados que se generan hasta la edad adulta y a la no identificación de factores de riesgo en el niño que puedan afectarlo en el futuro (CDC, 2003).

Hay que señalar que los estudios alrededor de los efectos de la actividad física formal sobre los aspectos fisiológicos en los niños son limitados debido a la dificultad de ajustar distintos protocolos experimentales en términos de equipamientos, procedimientos y test de medición que no se acomodan a las condiciones generales morfofisiológicas de los niños.

De todas maneras, los expertos del ejercicio deben profundizar sobre las bondades del ejercicio físico aplicado de manera sistemática, con el fin de desarrollar e instaurar características protectoras de la condición de salud y prevención de la enfermedad. Dentro de este objetivo principal se requiere tener un conocimiento suficiente sobre las principales características fisiológicas del niño y del adolescente deduciendo la respuesta fisiológica de los sistemas corporales al estrés fisiológico asociado al ejercicio, el desarrollo de las cualidades físicas básicas y perceptivomotoras, junto con la consolidación de los estadios psicosociales propios de esta parte del ciclo vital. El conocimiento científico de las respuestas fisiológicas del niño al ejercicio debe conducir a que todas las personas encargadas de la actividad física y el entrenamiento con niños deba diseñar planes de entrenamiento en coherencia con ellas.

\section{Características fisiológicas, alcances e implicaciones}

Para la prescripción del ejercicio físico en los niños y adolescentes es necesario conocer las características morfofisiológicas propias del mismo. La niñez se convierte en una etapa de falencias en términos de la manifestación de las cualidades físicas, sobre todo de la capacidad de resistencia anaeróbica, las cualidades coordinativas, de capacidad aeróbica, de fuerza y de adaptación al entorno.

Un aspecto fundamental que media esta expresión física es la composición ósea ya que está asociada al crecimiento del sujeto en términos de la talla, justo antes de la pubertad el ritmo de crecimiento se hace más lento permitiendo la iniciación en la maduración ósea y esquelética. El ejercicio físico en este sentido puede incrementar el grosor del hueso compacto y la densidad ósea permitiendo un depósito mayor de matriz ósea, lo que incrementa la ductilidad y la resistencia ósea (ACSM, 1990).

Un segundo aspecto fundamental que condiciona la respuesta metabólica y fisiológica en los niños es la actividad enzimática en los procesos exergónicos para la obtención de energía; junto con el desarrollo de la capacidad energética medida por los patrones de movilización y de producción energética.

Un tercer aspecto, es la poca información que existe sobre los fenómenos hormonales, celulares del músculo, al igual que los procesos que comprometen al sistema nervioso central o periférico, lo que limita la capacidad metabólica y de moviliza- 
ción de sustratos energéticos y su comprensión en la niñez. Lo que se conoce es que los niños presentan una vía anaeróbica restringida, una capacidad aeróbica limitada por factores morfológicos y fisiológicos como el tamaño del corazón y su volumen sistólico, la capilarización de los músculos, la concentración de mioglobina, entre otros (Berg, 1988).

Estas limitaciones afectan directamente los procesos de adaptación y respuesta fisiológica al ejercicio implicando la realización de éste de manera más lenta, prolongada y de menor intensidad, por lo menos mientras se alcanza la madurez biológica necesaria.

\section{Beneficios del ejercicio físico en niños y adolescentes}

La participación en programas de actividad física formal (ejercicio físico, deporte) promueven en términos generales una adecuada condición de salud. (USDHHS, 2002). Realizando un análisis comparativo de estudios realizados en niños activos con niños sedentarios se ha demostrado que los niños activos tienen niveles más bajos de presión arterial en reposo, niveles más bajos de lipoproteínas de densidad alta asociados a la actividad física regular (AHA,2003).

1. En este sentido, se puede afirmar que los niños que son hipocinéticos probablemente desarrollarán conductas de vida sedentarias, convirtiéndose en el futuro en un adulto sedentario, lo que deja entrever la importancia de fomentar la actividad física regular en los niños y adolescentes: como política pública local (USDHHS,1996), (CDC, USDHHS,1997),

Dentro de los beneficios cabe mencionar:

\section{Reducción del riesgo de enfermedades cardiovasculares}

Cada vez de manera más temprana los niños desarrollan factores de riesgo cardiovasculares como sedentarismo, sobrepeso, colesterol eleva- do en sangre y tabaquismo (Patrick, 2001). Cabe mencionar que los ataques cardiacos y accidentes cerebrovasculares son raros en los niños, pero esta condición de riesgo en la infancia condiciona la instauración de estos tipos de eventos patológicos en la adultez (AHA, 2003).

\section{Mantenimiento de un peso corporal saludable}

En el año 2000, se menciona que los niños y adolescentes estadounidenses entre los 6-19 años, triplicaron el porcentaje de obesidad y sobrepeso que los niños de los años ochenta (Sothern, 1999). Esta cifra es preocupante ya que los adolescentes con obesidad o sobrepeso son muy parecidos al adulto obeso, ya que presentan hiperlipidemia en sangre, hipertensión, aumento del porcentaje graso corporal, disminución en la manifestación de las cualidades físicas, disminución del tejido magro, problemas articulares y de postura (CDC, 2003). La actividad física regular en los niños y de manera continua en la adultez promueve el control y el mantenimiento de un porcentaje graso corporal menor $\mathrm{y}$ un aumento en la cantidad de tejido magro permitiendo mantener un peso saludable.

\section{Mantenimiento de una estructura osteomuscular saludable}

Se ha demostrado que la actividad física regular desde edades tempranas retarda en las mujeres hasta 10.4 años la aparición de los síntomas de osteoporosis, logrando un pico máximo de densidad ósea en la juventud, la cual se puede mantener, por más tiempo durante la adultez, lo que demuestra el efecto preventor que tiene el ejercicio. (CDC, 2004). La práctica de ejercicio físico aeróbico de naturaleza cíclica combinado con trabajo de musculación con carga libre favorece el mantenimiento de una adecuada densidad ósea.

\section{Mantenimiento de una salud mental y un bien- estar psicosocial}

Promueve salud psicológica y bienestar las presiones psicológicas y sociales, como las presiones 
y las preocupaciones sobre la apariencia, la imagen del cuerpo y peso que pueden ser significantes para los adolescentes, sobre todo en las mujeres. Los estudios han demostrado cómo la actividad física puede aumentar la autoestima de los adolescentes y puede reducir la ansiedad y el estrés. (Nurmi-Lawton, 2004).

\section{Desarrollo de destrezas motoras}

El ejercicio físico, permite la expresión de la capacidad cinética del niño, consolidando la instauración de las cualidades físicas básicas y permitiendo el desarrollo de las cualidades perceptimotoras. En este sentido el ejercicio físico se convierte en un potencializador de la capacidad de movimiento.

\section{Favorece la interacción social}

El ejercicio físico en el niño y el adolescente favorece la interacción social por su componente lúdico recreativo, el desempeño dentro de las actividades grupales de naturaleza formal, permite consolidar el desarrollo de la persona, y el juego de roles sociales.

\section{Evaluación del fitness}

La evaluación del fitness y la condición física en los niños son comunes dentro de la educación básica y media, particularmente dentro de la cátedra de educación física. En este sentido existe una serie de test y/o pruebas físicas que permiten medir el estado del fitness en los niños y adolescentes. Es fundamental para el experto en la prescripción del ejercicio conocer y aplicar esta serie de instrumentos de medida del fitness, con el fin de realizar un diagnóstico del estado del sujeto en términos de su condición física y de salud. La evaluación del fitness permite medir la capacidad de trabajo físico de acuerdo con el funcionamiento fisiológico, permite identificar las deficiencias de los componentes del fitness específico, evalúa la eficien- cia de un programa de prescripción del ejercicio (Sothern, 1999).

A partir de la Segunda Guerra Mundial toma importancia la valoración de cada componente de la condición física. En 1958, la Asociación Americana para la Salud, la Educación Física y la Recreación, intenta unificar criterios de valoración de cada cualidad en función de la edad, proponiendo la evaluación de la fuerza de la extremidad superior (tracción de brazos), la resistencia muscular abdominal (abdominales con las piernas flexionadas), la agilidad (carrera de ida y vuelta sobre un trazado de 10 yardas), la potencia de la extremidad inferior (salto horizontal a pies juntos), la velocidad de desplazamiento (50 yardas), la resistencia cardiovascular (600) yardas, 9 minutos de carrera ó 12 minutos de carrera).

A partir de este momento se han desarrollado una serie de test y baterías de evaluación. como la International Committee for Standardization of Physical Fitness Tests (ICSPFT, 1974). Fleischmann Physical Fitness Test (Fleischmann. 1964) Leuven Growth Study (Ostyn y otros, 1980: Hebbelinck y otros, 1980; Beunen y otros, 1983; Hebbelinck y Borms, 1969, 1973). Test de Condition Moper (Kemper, 1981) el Test de Condition motrice pour les écoles finlandaises (Telama, Nuppanen y Holopainen, 1983), entre otras.

En el seno del Consejo de Europa para la valoración de la condición física en 1977, en un comité de expertos se unificó una batería para Europa de evaluación de la condición física denominada "Eurofit" (Harris, 2006).

En la actualidad existe una serie de baterías de campo de fácil aplicación, las cuales contienen 4 a 6 pruebas, que permiten medir el estado del fitness físico en los niños y adolescentes, entre los que se puede mencionar se encuentra el test denominado "Fitnessgram". Este test fue desarrollado por el Cooper Institute for Aerobics Research. Dallas, Texas, en donde se evalúa la capacidad aeróbica, 
la composición corporal, la fuerza de resistencia y la flexibilidad (tabla 1). (Yucra, 2001).

\section{TABLA 1 \\ ÍtemES DEL TEST FITNESSGRAM}

\section{Capacidad aeróbica}

- El PACER - recomendado para estudiantes de nivel preescolar a 3 grado (varias fases de 20 metros de "Shuttle run").

- Una milla caminando/corriendo.

\section{Composición corporal}

- Porcentaje de grasa - calculado de los tríceps y de los pliegues de piel de las pantorrillas.

- Índice de la masa del cuerpo - calculado por el peso y estatura.

\section{Fuerza muscular, resistencia, y flexibilidad}

\section{Fuerza abdominal}

- Curl-up test.

\section{Extensores de tronco (fuerza y flexibilidad)}

- Trunk lift (elevación de tronco).

Fuerza muscular de la parte superior del cuerpo

- Push-up.

- Pull-up modificado.

- Pull-up.

- Flexed arm hang (suspensión con brazos flexionados).

\section{Flexibilidad}

- Back-saver-Sit-and-reach (sentarse y alcanzar).

- Shoulder stretch (extensión de hombros).

Fuente bibliográfica: Cooper Institute for Aerobics Research (1994). The Prudential Fitness gram: Test administration manual. Dallas: Texas.

Asimismo, el Centro de Estudios de Laboratorio de la Aptitud Física de São Paulo, del sur

CELAFISC en Brasil, propuso una batería de evaluación para la medición de la aptitud física, teniendo en cuenta aspectos antropométricos, metabólicos, neuromusculares y de maduración biológica y psicosocial (tabla 2 ).

\section{TABLA 2 \\ ÍTEMES DE EVALUACIÓN DE APTITUD FÍSICA (CELAFISC):}

\section{Antropométricas \\ a. Peso \\ b. Altura \\ encefálica \\ c. Composición corporal}

\section{Metabólicas}

a. Potencia aeróbica $\left(\mathrm{VO}_{2}\right.$

b. Potencia anaeróbica

\section{Neuromusculares}

a. Fuerza de miembros inferiores

b. Fuerza de miembros

Superiores

Dinamométrica

c. Velocidad

d. Agilidad

\section{Psicosocial}
a. Psicológicas
b. Sociales
a. Percepción del esfuerzo
b. Sociometría

a. Test de salto vertical - Con ayuda de los brazos - Sin ayuda de los brazos Test de salto horizontal

b. Test de prensión manual:

c. Test carrera de $50 \mathrm{~m}$

d. Test de Shuttle Run

\section{Maduración biológica}

\begin{tabular}{ll}
$\begin{array}{l}\text { a. Sexo femenino } \\
\text { b. Sexo masculino }\end{array}$ & a. Edad de menarquia \\
axilar & b. Evaluación del pelo \\
c. Ambos sexos & c. Autoevaluación de las \\
& $\begin{array}{l}\text { características sexuales } \\
\text { secundarias }\end{array}$ \\
\hline
\end{tabular}

Fuente: www.celafisc.com.br
a. Peso
c. Pliegues cutáneos
Circunferencias musculares Diámetros óseos
a. Consumo de oxígeno
b. Test carrera de $40 \mathrm{seg}$. 
Además de lo anterior, existe una serie de test para la valoración individualizada de los componentes del fitness y la condición física en los niños, por ejemplo el test de Gallager y Bronha y el test de Ruffier y Dickson que sirven para medir la adaptación y el coeficiente de resistencia cardiovascular, los cuales son métodos indirectos para la evaluación del VO2 máximo. Por otro lado, en cuanto a la evaluación de la fuerza se han propuesto test de campo como pruebas de salto vertical u horizontal, pruebas de dinamometría para valoración del fitness muscular, test de resistencia flexiones de brazo, test de fuerza abdominal. entre otros. (US Department of Health and Human Services, 1996).

De la misma manera, existe literatura de una serie de instrumentos apropiados para medir otros indicadores morfofisiológicos en los niños que se utilizan por su valor predictivo o estimativo útiles en el tamizaje de grandes grupos poblacionales como son los cuestionarios de autocalificación, los sensores de movimiento que son confiables para la determinación del nivel de actividad física (Heyward, 2001).

\section{Recomendaciones para la prescripción del ejercicio}

Los niños y adolescentes dejan de ser activos a medida que van creciendo, declinando su nivel de actividad física. Por tanto, si se logra mantener niveles altos de actividad física durante la infancia y la adolescencia se podrá mantener estilos de vida activos y conductas saludables en la adultez.

En este sentido, muchos son partidarios de implementar las mismas pautas de prescripción del ejercicio que se utilizan en el adulto sedentario en los niños. Aunque esta posición todavía no tenga un soporte científico suficiente es importante retomar como punto de referencia los principios generales de la prescripción del ejercicio en términos de la frecuencia, la intensidad. del tipo de ejercicio, la progresión y la duración se- gún ACSM para la prescripción en los niños. (ACSM, 1999).

En términos generales el ejercicio físico recomendado para esta población y su prescripción se centra más en el gasto de energía. que en sí, en la aplicación de cargas de trabajo con intensidades y volúmenes sistemáticos de entrenamiento. La cantidad óptima y el tipo de ejercicio físico que se debe prescribir no están totalmente definidos, lo que se recomienda es que el niño o adolescente combine actividades físicas formales y no formales que permitan una actividad cinética importante y por consiguiente un gasto energético mayor, supeditado al nivel de madurativo, de crecimiento, de destreza motora y de experiencias motoras previas en el niño. (ACSM, 2000).

Asimismo, la prescripción del ejercicio se debe iniciar después de los 6 años, una vez se completa la instauración de los patrones motores básicos de locomoción, acumulando mínimo 30 minutos diarios de ejercicio moderado, siendo preferible su realización diaria. (Pate, 1995).

Sin embargo, cabe mencionar que se pueden realizar actividades de intensidad alta tres veces a la semana, trayendo consigo los mismos beneficios que el ejercicio de intensidad moderada pero realizado diariamente. (US Department of Health and Human Services. 1996).

Otro aspecto a tener en cuenta en la elaboración de programas de ejercicio físico para niños y adolescentes es el fuerte componente lúdico-recreativo que deben tener dichos programas y la realización de actividades socializadoras. En la edad preescolar, se debe realizar una gran variedad de actividades físicas que favorezcan el esquema corporal, el juego, la coordinación. En los niños prepuberales se deben proponer actividades físicas más formales, el aprendizaje de gesto complejos de movimiento, el trabajo aeróbico ligero y el mantenimiento de la flexibilidad, involucrando, contracciones dinámicas y ejercicios de naturale- 
za rítmica que contribuyan a la maduración biopsicosocial del niño.

\section{Consideraciones para el entrenamiento de la capacidad aeróbica}

El desarrollo de la capacidad aeróbica no es un proceso lineal, los niños y adolescentes poseen un VO2 máx. menor entre un 15 a $25 \%$ comparado con los adultos, (Wilmore, 2004). Los niños y adolescentes, utilizan frecuencias cardiacas mayores que la de los adultos durante esfuerzos máximos y submáximos, lo que impacta en su capacidad de mantener un ritmo sostenido de trabajo cardiaco. (Rowland, 1988). Las ganancias funcionales en el consumo de oxígeno se logran después de entrenamientos entre 10 a 12 semanas a un 60 a $70 \%$ del VO2 máx., este tipo de entrenamiento, con el objetivo de mejorar el consumo de oxígeno no debe iniciarse antes de los 12 años; sin embargo, otros autores encuentran mejorías sustanciales de la capacidad anaeróbica después de entrenamientos de alta intensidad en niños deportistas de élite, de cualquier forma la recomendación es esperar el total de la maduración y completo desarrollo para iniciar entrenamientos encaminados a mejorar esta cualidad. (Daniela, 1971).

Para mejorar la capacidad y potencia aeróbica se utilizan diversos métodos de entrenamiento, entre los cuales se pueden mencionar métodos de entrenamiento continuo o intervalado; en los niños se recomiendan métodos de entrenamiento continuo con trabajo aeróbico ligero no mayor de 30 minutos de actividad, ya que los niños no pueden ajustar fácilmente sus mecanismos de termorregulación, lo que puede generar desmayos, pérdidas de conciencia, deshidratación fácilmente. (McArdle, 1981).

Desde otra especificidad, la identificación de la zona de entrenamiento debe ser delimitada a través de la aplicación de la escala del esfuerzo percibido (EFP) o a través de la determinación del VO2 máx., no se recomienda la prescripción de trabajo aeróbico con base en la frecuencia cardiaca de reserva ya que los niños tienen un gasto cardiaco bajo, debido al tamaño menor del corazón, al menor volumen sistólico (Rowland, 1985). La intensidad del ejercicio debe ser moderada, entre 40 a $85 \%$ del VO2 de reserva (Bar-Or, O., 2001).

La duración del tiempo del ejercicio en niños y adolescentes se hace con base al gasto calórico, el cual en niños prepuberales se recomienda gastar entre 200 y 250 calorías por sesión de trabajo. Se recomienda un tiempo de calentamiento largo, permitiendo los ajustes fisiológicos y la respuesta al ejercicio adecuadamente y estable, lo ideal es que ese calentamiento sea de 15 minutos, fase central de 30 minutos y el regreso a la calma de 5 a 10 minutos respectivamente.

\section{Consideraciones para el entrenamiento de la fuerza}

La American Academic of Pediatrics (AAP, 2001), realizó una serie de recomendaciones sobre el entrenamiento de la fuerza en niños y adolescentes debido a que este es un componente común de los programas de actividad física para la población infantil. Algunos adolescentes y preadolescentes utilizan estos programas como un medio para mejorar el tamaño muscular o para mejorar la apariencia física. Estos programas incluyen el uso de pesos libres, pesos con máquinas, tubos elásticos o ejercicios, el cual debe ser dosificado de manera adecuada. La cantidad y la forma de resistencia muscular utilizada así como también la frecuencia están determinadas por las metas específicas del programa, en términos de ayudar a mantener una musculatura adecuada.

Se debe iniciar con poco peso para mejorar primero la ejecución del movimiento. Para obtener ganancias en la fuerza es necesario realizar programas de musculación que incluye entre 8-10 ejercicios diferentes que involucren los grandes grupos musculares, cada uno debe realizarse entre 10 
a 15 repeticiones y trabajar dentro de una es la de calificación de 12-13 de la EEP. El incremento de la carga de trabajo debe realizarse a expensas en el aumento de las repeticiones más no del aumento de la carga durante 20 a 30 minutos de 2 a 3 veces por semana. (Falk, 1996).

Estudios han mostrado que el entrenamiento de la fuerza dentro de un programa general de prescripción estructurada con propiedad puede incrementar la fuerza en preadolescentes y adolescentes. Las ganancias en la fuerza, la potencia y tamaño muscular se consiguen a partir de 6 semanas de entrenamiento continuo, aunque no se puede esperar grandes resultados en el proceso de hipertrofia. En preadolescentes el aumento de la fuerza no se da necesariamente con la hipertrofia muscular. Las ganancias en la fuerza pueden deberse al aprendizaje neuromuscular incrementándose el número de motoneuronas que son utilizadas durante la contracción muscular. Este mecanismo permite explicar la fuerza obtenida del entrenamiento de resistencia en poblaciones con bajos niveles de andrógenos, incluyendo mujeres y preadolescentes masculinos. El entrenamiento de la fuerza puede también aumentar el alargamiento que normalmente ocurre con el crecimiento durante la pubertad en hombres y mujeres. La evidencia de que los programas de fuerza pueden reducir el riesgo de lesión en adolescentes y preadolescente no es concluyente. (Ramsay, 1990), (Blimkie, 1993) (Faigenbaum, 1993) (Drinkwater, 1977).

Los programas adecuados de entrenamiento de fuerza deben centrarse en la ganancia del equilibrio muscular, la flexibilidad, la técnica apropiada y el mantenimiento de una adecuada masa muscular. (Drinkwater, 1979).

\section{Consideraciones para el entrenamiento de la flexibilidad}

Existe una tendencia natural de disminuir los grados de flexibilidad en los adolescentes produc- to del proceso de crecimiento y desarrollo, el objetivo principal de la prescripción de la flexibilidad es mantener esos niveles adecuados de flexibilidad con el fin de prevenir lesiones musculares, malos alineamientos de segmentos corporales, problemas de espalda, dolor articular, entre otros. Un programa de estiramiento debe ser ejecutado de 2 a 3 veces a la semana incluyendo ejercicio de estiramiento para cada grupo muscular con una duración de 10 a $30 \mathrm{sg}$ dentro de cada sesión de trabajo, en donde el estiramiento debe realizarse de manera lenta y sostenida.

\section{Precauciones durante la ejecución del ejercicio}

Uno de los principales aspectos a tener en cuenta es el tema del entorno ambiental, la humedad. la temperatura ambiente que condicionan el buen desempeño en la hora de realizar ejercicio físico en ambientes naturales, esto debido a que los niños tienen menos superficie corporal. lo que implica una menor capacidad para disipar el calor, además de tener un metabolismo mayor.

Bajo las anteriores condiciones, recomendado no realizar actividad física al aire libre cuando la temperatura ambiente sea mayor de $35^{\circ}$ ya que bajo estas condiciones ambientales se puede generar estrés fisiológico por calor. (Wagner, 1972).

Con base a las siguientes afirmaciones la American Academy of Pediatrics, 2000 recomienda:

Se deben tener en cuenta los procesos de aclimatización de 10 a 14 días en los niños cuando el ejercicio físico que se prescribe se realice en ambientes cálidos.

Ante una práctica prolongada de ejercicio el niño debe hidratarse cada 20 minutos ingiriendo $159 \mathrm{ml}$ de agua ( 5 onzas) si el niño pesa menos de $40 \mathrm{~kg}$ y $250 \mathrm{ml}$ de agua si pesa menos de $60 \mathrm{~kg}$.

Antes y después de la realización del ejercicio se deben verificar los cambios en el peso 
corporal y la presencia o no de síntomas de deshidratación.

El ejercicio debe realizarse con ropa cómoda que permita la fácil dispersión del calor y la evaporización con el fin de favorecer los mecanismos termorregulatorios del niño.

Con las anteriores evidencias científicas se pretende argumentar la importancia que debe tener para el fisioterapeuta la prescripción adecuada de ejercicio físico en los niños y adolescentes de manera regular. Así entre más activo sea el niño y/o el adolescente se podrá favorecer una mejor adaptación biológica, el desarrollo y potencialización de las cualidades físicas básicas y en general la capacidad cinética de los niños y adolescentes de una manera adecuada y segura.

Recibido : octubre 2006 Aceptado: octubre 2006

\section{Referencias bibliográficas}

ACSM (1999). Manual para la valoración y la prescripción del ejercicio. Barcelona, Paidotribo.

ACSM (2000). Manual de consulta para el control y la prescripción del ejercicio". Barcelona, Paidotribo.

American College of Sports Medicine (1990). The recommended quantity and quality of exercise developing an maintaining cardio respiratory and muscular fitness in healthy adults. Med Sci Sports Exerc, 22: 265-274.

American Heart Association AHA, Children's Need for Physical Activity: Fact Sheet. Accessed June 2003 (www.americanheart.org)

American Academy of Pediatrics (2001). Committee on Sports Medicine and Fitness Strength Training by Children and Adolescents.

BAR-Or O (1989). Trainability of the prepubescent child. Phys. Sportsmed, 17: 65-81.

Berg A and Keul J (1988). Biochemical change during exercise in children. In: Malina, RM (ed.). Young Athletes. Biological, Psycho- logical, and Educational Perspectives, 6177. Champaign, IL: Human Kinetics.

Blimkie CJ (1993). Sports Med, 15: 389-407.

CDC, USDHHS. MMWR March 7, 1997 Guidelines for School and Community Programs to Promote Lifelong Physical Activity Among Young People.

Centers for Disease Control and Prevention, U.S. Department of Health and Human Services, Public Health Service. Morbidity and Mortality Weekly Report (MMWR) October 26, 2001, vol. 50, No. RR-18. Increasing Physical Activity - A Report on Recommendations of the Task Force on Community Preventive Services. Also published in American Journal of Prevention Medicine 2002; 22 (4S), 67-72.

Centers for Disease Control and Prevention (CDC), National Center for Chronic Disease Prevention and Health Promotion, Nutrition and Physical Activity - National Bone Health Campaign. Accessed in August 2003.

Centers for Disease Control and Prevention (CDC) and President's Council on Physical Fitness and Sports. Healthy People 2010. Leading Health Indicators.

Daniels J and Oldridge N (1971). Changes in oxygen consumption in young boys during growth and running training. Med Sci Sports, 3: $161-165$.

Drinkwater BL, Kupprat IC, Denton JE, et al. (1977). Response of prepubertal girls and college women to work in the heat. J Appl Physiol; 43.

Drinkwater BL, Horvath SM (1979). Heat tolerance and aging. Med Sci Sports Exerc, 11: 4955 .

Falk B, Tenenbaum G (1996). The effectiveness of resistance training in children. A metaanalysis. Sports Med, 3: 176-186.

Faigenbaum AD, Zaichiowsky LD, Westcott WL, Micheli LJ, Fehlandt AF (1993). The effects of a twice-a-week strength training program on children. Pediatr Exerc Sci, 5: 339-346. 
Harris Jo, Cale Lorraine (2006). A review of children's fitness testing. European Physical Education Review, 12: 201-225.

Heyward, Vivian H (2001). Evaluación y prescripción del ejercicio. Valoración de la fuerza y de la resistencia muscular. Cap. 4, 2a. ed.. Ed. España. Paidotribo.

McArdle: WD, Katch FI and Katch VL (1981). Exercise physiology: energy nutrition, and human performance. Philadelphia: Lea \& Febiger.

Nurmi-Lawton Ja. Baxter-Jones A. Taylor P. Cooper C. Bishop J, New S (2004). Dietary balance in physically active and in-active girls, in Nutritional aspects of osteoporosis, Burckhardt P. Dawson-Hughes B. Heaney RP (eds.), San Diego: Elsevier Science. Chapter 31: 411-418.

Patrick K, Spear B, Holt K. Sofka D, (eds.) (2001). Bright futures in practice: physical activity: Arlington. VA: National Center for Education in Maternal and Child Health.

Pate RR. Smal. ML, Ross JG. Young JC. Flint KH \& W ARREN CW (1995). School physical education. Journal of School Health, 65 (8): 339-343. EJ 520865.

Promotion and the President's Council on Physical Fitness and Sports (PCPFS). Physical Activity and Health - A Report of the Surgeon General, 1996.

Ramsay Ja, Blimkie CJ. Smith K. Garner S, MacDougall. J. Sale DG (1990). Strength training effects in prepubescent boys. Issues and controversies. Med Sci Sports Exerc. 22: 605-614.

Rowland TW (1990). Exercise and Children's Health. Champaign, I.L: Human Kinetics.

RoWl.AND TW (1985). Aerobic response to endurance training in prepubescent children: a critical analysis. Med Sci Sports Exerc, 17: 493-497.

Rowland TW, StaAb J, Unnithan V and Siconolffi S (1988). Maximal cardiac responses in prepubertal and adult male (abstract). Med Sci Sports Exerc 20 (Suppl.): S32.

Sallis JF, Prochaska JJ and Taylor WC. (2000). A review of correlates of physical activity of children and adolescents. Med Sci Sports Exerc, vol. 32. No. 5, 963-975.

Sothern MS, Loftin M. Siskind RM, Udall JN, BLECKER U (1999). The health benefits of physical activity in children and adolescents: implications for chronic disease prevention. European Journal of Pediatrics, 158: 271274.

The Cooper Institute for Aerobics Research. (1999). FITNESSGRAM test administration manual, Champaign, IL: Human Kinetics. The President's Council on Physical Fitness and Sports (PCPFS).

US Department of Health and Human Services (USDHHS), Centers for Disease Control and Prevention (CDC), National Center for Chronic Disease Prevention and Health Promotion and The President's Council on Physical Fitness and Sports (PCPFS). Phisical Activity and Health - A Report of the Surgeon General. 1996.

US Department of Health and Human Services (USDHHS). Office of the Assistance Secretary for Planning and Evaluation. Physical Activity Fundamental to Preventing Disease. June 20. 2002.

USDHHS Physical Activity Fundamental to Preventing Disease (2002).

Waginer JA. Robinson S, TZankoff SP et al. (1972). Heat tolerance and acclimatization work in the heat in relation to age. J Appl Physiol, 33: 616-622.

Wilmore JH, Costill. DL (2004). Fisiología del ejercicio y del deporte. Barcelona: Ed. Paidotribo.

YLCRA (2001). Algunas consideraciones para la utilización de las baterías de test de la condición física http://www.efdeportes.com/ Revista Digital - Buenos Aires - Año 7 - N 38. julio. 